\title{
STABILITY OF THE TRIANGULAR LAGRANGIAN SOLUTIONS OF THE PHOTO GRAVITATIONAL RESTRICTED THREE-BODY PROBLEM IN THE Three-Dimensional CASE
}

\author{
Md. Ghulam Murtuza \\ Research Service Centre, Bhagalpur University \\ Vijay Kumar and R.K. Choudhry \\ Dept. of Maths., Bhagalpur University
}

\begin{abstract}
The stability of the triangular Lagrangian solutions for the photo-gravitational restricted three-body problem in the three-dimensional case is investigated for the case when the resonances are absent and also when the resonances are present. Stability is proved for most (in the sense of Lebesgue) initial conditions for all $\mu<\mu_{0}$ except for the resonance cases.
\end{abstract}

\section{INTRODUCTIION}

This work is a general isation over the work by Kumar and Choudhry $(1987,1988)$ in the sense that here we have taken up three-dimensional case and a generalisation over Markeev's work (1972) in the sense that here we have taken photo-gravitational effects of the two finites bodies which have been assumed to be radiating ones as well.

Here we have studied the stability of the triangular Lagrangian solutions. Since we have two such solutions situated symmetrically, so we have taken up the study of the stability of $L_{4}$ alone and it is claimed that the nature of $L_{5}$ will be the same.

For the investigation of the stability we have tried to reduce our Hamiltonian to a form suitable for the application of Arnold-theory (1963). The equations of motion have been normalised by Birkhoff's transformations. The non-resonace case has been dealt in section 4 and the resonance cases in section 5. The resonance case when $\omega_{1}=\omega_{2}$ has not been taken up. 


\section{THE HAMILTONIAN OF THE PERTURBED MOTION}

We introduce the rotating coordinate system $(0, x y z)$. Its origin coincides with 0 , the centre of mass of the bodies $p_{1}$ and $\mathrm{P}_{2}$, its $\mathrm{Ox}$-axis is directed towards the body $\mathrm{p}_{2}$, the $\mathrm{Oy}_{-}$ axis lies in the rotation plane of the bodies $P_{1}$ and $P_{2}$ perpendicular to $P_{1} P_{2}$ and the $O z$-axis completes a $r$ ight-handed coordinate system with $O x$ and Oy. We adopt the units of measurement for $t$ ime and length such that the angular rotational velocity of the bodies $P_{1}$ and $P_{2}$ and the distance between them are equal to unity. The sum of masses of $P_{1}$ and $P_{2}$ will be taken for unit mass. Both of the bodies $\vec{P}_{1}$ and $\vec{P}_{2}$ are taken to be radiating and the reduction factors $\alpha$ and $\beta$ are assumed to be such that $0<\alpha, \beta<1$ as detailed in the work (1987).

If $x, y, z$ be the coordinates of the body $P$ and $P_{x}, p_{y}, p_{z}$ the corresponding momenta, then the Hamilton function for the photo-gravitational restricted three body problem will take the form:

$$
\begin{aligned}
& H=\frac{1}{2}\left(P_{x}^{2}+P_{y}^{2}+P_{z}^{2}\right)+P_{x} y-P_{y} x-\alpha(1-\mu) / P_{1} P-\beta \mu / P_{z} P \\
& P_{1} p^{2}=(x+\mu)^{2}+y^{2}+z^{2} \\
& P_{2} p^{2}=(x-1+\mu)^{2}+y^{2}+z^{2} .
\end{aligned}
$$

In the $(0, x y z)$ coordinate system the triangular solution corresponding to the equilibrium position $L_{4}$ is given as

$$
\begin{aligned}
& \mathrm{x}_{\mathrm{o}}=\frac{\delta_{1}^{2}+1-\delta_{2}^{2}}{2}-\mu, \mathrm{y}_{\mathrm{o}}=\delta_{1} \delta_{2} \sqrt{\mathrm{b}}, \quad \mathrm{z}_{\mathrm{o}}=0 \\
& \mathrm{Px}_{\mathrm{o}}=-\delta_{1} \delta_{2} \sqrt{\mathrm{b}} \quad, \mathrm{Py}_{\mathrm{o}}=\frac{\delta_{1}^{2+1}-\delta_{2}^{2}}{2}-\mu, \mathrm{Px}_{\mathrm{o}}=0 \\
& \alpha=\delta_{1}^{3}, \quad \beta=\delta_{2}^{3}, \\
& \mathrm{~b}=1-\frac{\left(\delta_{1}^{2}+\delta_{2}^{2}-1\right)^{2}}{4 \delta_{1}^{2} \delta_{2}^{2}}
\end{aligned}
$$

If we make the change of variables 


$$
\begin{aligned}
x & =x_{0}+q_{1}, \quad y=y_{0}+q_{2}, \quad z=z_{0}+q_{3} \\
p_{x} & =p_{x_{0}}+p_{1}, \quad p_{y}=p_{y_{0}}+p_{2}, \quad p_{z}=p_{z_{0}}+p_{3},
\end{aligned}
$$

the motion in question will correspond to the equilibrium position $\mathrm{q}_{1}=\mathrm{p}_{1}=0(i=1,2,3)$.

The Hamiltonian function (i) may be expanded in the neighbourhood of the equilibrium position as

$$
\mathrm{H}=\mathrm{H}_{2}+\mathrm{H}_{3}+\mathrm{H}_{4}+\ldots+\mathrm{H}_{\mathrm{m}}+\ldots
$$

where $H_{m}$ is a homogeneous function of degree $m$ with respect to $q_{1}$ and $p_{1}$. In particular,

$$
\begin{aligned}
\mathrm{H}_{2}= & (1 / 2)\left(p_{1}^{2}+p_{2}^{2}+p_{3}^{2}\right)+p_{1} q_{2}-p_{2} q_{1}+q_{1}^{2}[1-(3 / 4)\{(1-\mu) \times \\
& \left.\left.\xi^{2} \delta_{1}^{-2}+\mu \eta^{2} \delta_{2}^{-2}\right\}\right] / 2-(3 / 2) q_{1} q_{2} \sqrt{b / \delta} \delta_{1} \delta_{2}\left(\delta_{2}^{2}(1-\mu) \xi+\delta_{1}^{2} \mu \eta\right\} \\
& -(1 / 2) q_{2}^{2}\left[3 b\left[(1-\mu) \delta_{2}^{2}+\mu \delta_{1}^{2}\right\}-1\right]+(1 / 2) q_{3}^{2} \\
H_{3}= & (1 / 16) q_{1}^{3}\left[(1-\mu) \xi \delta_{1}^{-4}\left(5 \xi^{2}-12 \delta_{1}^{2}\right)+\mu \eta \delta_{2}^{4}\left(5 \eta^{2}-12 \delta_{2}^{2}\right)\right] \\
& +(3 / 8) q_{1}^{2} q_{2} / \bar{b}\left[(1-\mu) \delta_{2} \delta_{1}^{-3}\left(5 \xi^{2}-4 \delta_{1}^{2}\right)+\mu \delta_{1} \delta_{2}^{-3}\left(5 \eta^{2}-4 \delta_{2}^{2}\right)\right] \\
& +(3 / 4) q_{1} q_{2}^{2}\left[\xi \delta_{1}^{-2}(1-\mu)\left(5 b \delta_{2}^{2}-1\right)+\eta \delta_{2}^{-2} \mu\left(5 b \delta_{1}^{2}-1\right)\right]+ \\
& +q_{2}^{3} / \bar{b}\left[(1-\mu) \delta_{2} \delta_{1}^{-1}\left(5 \delta_{2}^{2} b-3\right)+\mu \delta_{1} \delta_{2}^{-1}\left(5 \delta_{1}^{2} b-3\right)\right] / 2 \\
& -(3 / 4) q_{1} q_{3}^{2}\left[(1-\mu) \xi \delta_{1}^{-2}+\mu \eta \delta_{2}^{-2}-(3 / 2) q_{2} q_{3}^{-2} / \bar{b}\left[(1-\mu) \delta_{2} \delta_{1}^{-1}\right.\right. \\
& \left.+\mu \delta_{1} \delta_{2}^{-1}\right]
\end{aligned}
$$$$
\mathrm{H}_{4}=-(1 / 8) \mathrm{q}_{1}^{4}\left[(1-\mu) \delta_{1}^{-6}\left\{3 \delta_{1}^{4}-(15 / 2) \xi^{2} \delta_{1}^{2}+(35 / 16) \xi^{4}\right\}\right.
$$$$
\left.+\mu \delta_{2}^{-6}\left\{3 \delta_{2}^{4}-(15 / 2) \eta^{2} \delta_{2}^{2}+(35 / 16) \eta^{4}\right\}\right]
$$$$
+(5 / 4) q_{1}^{3} q_{2} / \bar{b}\left[(1-\mu) \delta_{2} \delta_{1}^{-3} \xi\left\{3-(7 / 4) \xi^{2} \delta_{1}^{-2}\right\}\right.
$$ 
$\left.+\delta_{1} \delta_{2}^{-3} n\left\{3-(7 / 4) n^{2} \delta_{2}^{-2}\right\}\right]$

$+(5 / 4) q_{1} q_{2}^{3} / b\left[(1-\mu) \xi \delta_{2} \delta_{1}^{-3}\left(3-7 \delta_{2}^{2} b\right)+\mu n \delta_{1} \delta_{2}^{-3}\left(3-7 \delta_{1}^{2} b\right)\right]+$

$+(3 / 4) q_{1}^{2} q_{1}^{2}\left[(1-\mu) \delta_{1}^{-2}\left(-1+5 \delta_{2}^{2} b+(5 / 4) \xi^{2} \delta_{1}^{-2}\right.\right.$

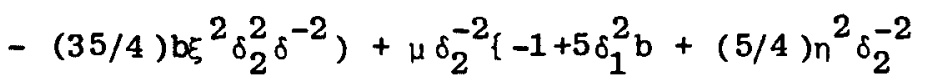

- $\left.\left.(35 / 4) \eta^{2} \delta_{1}^{2} b_{2}^{-2}\right\}\right]-(1 / 8) q_{2}^{4}\left[(1-\mu) \delta_{1}^{-2}\left(3-30 b \delta_{2}^{2}+35 \delta_{2}^{4} b^{2}\right)\right.$

$\left.+\mu \delta_{2}^{-2}\left(3-30 \mathrm{~b} \delta_{1}^{2}+35 \delta_{1}^{4} \mathrm{~b}^{2}\right)\right]$

$+(3 / 16) q_{1}^{2} q_{3}^{2}\left[-4(1-\mu) \delta_{1}^{-2}-4 \mu \delta_{2}^{-2}+5(1-\mu) \xi^{2} \delta_{1}^{-5}+\right.$

$\left.+5 \mu n^{2} \delta_{2}^{-5}\right]-(3 / 4) q_{2}^{2} q_{3}^{2}\left[(1-\mu) \delta_{1}^{-2}\left(1-5 \delta_{2}^{2} b\right)+\right.$

$+\mu \delta_{2}^{-2}\left(1-5 \delta_{1}^{2} b\right)$

$+(15 / 4) r b q_{1}{ }_{2} q_{3}^{2}\left[(1-\mu) \xi \delta_{2} \delta_{1}^{-3}+\mu \eta \delta_{1} \delta_{2}^{-3}\right]$

- $(3 / 8) q_{3}^{4}\left[(1-\mu) \delta_{1}^{-2}+\mu \delta_{2}^{-2}\right]$

where $\xi=\delta_{1}^{2}+1-\delta_{2}^{2}, n=\delta_{1}^{2}-1-\delta_{2}^{2}$.

3. CHARACTERISTIC ROOTS AND THE FIRST ORDER STABILITY OF THE TR IANGULAR LI IBERATION POINTS

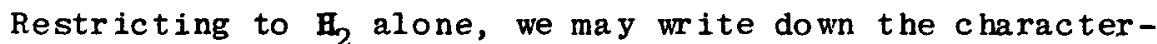
istic equation in the form

$$
\left[\lambda^{4}+\lambda^{2}+9 \mu(1-\mu) b\right]\left(\lambda^{2}+1\right)=0
$$

As in the planar case (1987) the value $\mu=0.285954=\mu_{0}$ (say) for $b=1$ corresponds to a critical case which needs special consideration and we shall not take it up here. We shall investigate the stability for all admissible values of b for $\mu<\mu_{0}$, where $\mu_{0}$ is given by Table-I (1987).

If $\omega_{1}, w_{2}$ and $w_{3}$ be the frequencies, then 
IABLE

III

\begin{tabular}{|c|c|c|c|c|c|c|}
\hline$b=$ & $\omega_{1}-\omega_{2}=0$ & $\omega_{1}-2 \omega_{2}=0$ & $\omega_{1}-3 \omega_{2}=0$ & $2 \omega_{2}-\omega_{3}=0$ & $3 \omega_{2}-\omega_{3}=0$ & $2 \omega_{1}-\omega_{2}-2 \omega_{3}=0$ \\
\hline 0.00 & not appiceble & N.A & M.A & N.A & M.A & N.A \\
\hline 0.05 & laaginary & Imaginary & 02763932 & 0.160666 & Inaginary & \\
\hline 0.10 & I maginery & 0.231251 & 0.1122701 & 0.0750100 & Imaginery & ineginery \\
\hline 0.15 & 0.2456126 & 0.1373962 & 0.07112255 & 0.068666 & Imaginery & I meginery \\
\hline 0.20 & 0.1666666 & 0.0906135 & 0.05271064 & 0.036019 & 0.325290 & Imaginary \\
\hline 0.25 & 0.127322 & 0.0770676 & 0.0617626 & 0.021595 & 0.227213 & Imaginery \\
\hline 0.30 & 0.1032539 & 0.632612 & 0.0365253 & 0.023110 & 0.171004 & Inaginary \\
\hline 0.35 & 0.6869102 & 0.0536766 & 0.0296310 & 0.020250 & 0.167036 & Inaginary \\
\hline 0.40 & 0.0750011 & 0.666176 & 0.0756503 & 0.017673 & 0125680 & 0.622560 \\
\hline 065 & 0.0660912 & 0.0412039 & 0.0227392 & 0.015671 & 0.109560 & 0.350186 \\
\hline 0.50 & 0.0590616 & 0.0369185 & 0.0206160 & 0.016010 & 0.091260 & 0.287397 \\
\hline 0.55 & 0.0533516 & 0.0336615 & 0.0115269 & 0.0127700 & 0.087450 & 0.267373 \\
\hline 0.60 & 0.0636665 & 0.0305637 & 0.0169541 & 0.011710 & 0.079670 & 0.211320 \\
\hline 0.63 & 0.166363 & 0.02101626 & 0.0156201 & 0.010100 & 0.072030 & 0.195910 \\
\hline 0.70 & 0.0613961 & 0.0260768 & 0.0164951 & 0.010021 & 0.067120 & 0.177960 \\
\hline 0.75 & 0.0315208 & 0.0262938 & 0.0135160 & 0.009366 & 0.062620 & 0.163140 \\
\hline 0.10 & 0.0360190 & 0.227392 & 0.0126602 & 0.000757 & 0.051260 & 0.150710 \\
\hline 0.85 & 0.0338237 & 0.0213717 & 0.0119066 & 0.001237 & 0.056620 & 0.140090 \\
\hline 0.90 & 0.0310105 & 0.0207596 & 0.0112373 & 0.007178 & 0.051610 & 0.130910 \\
\hline 0.95 & 0.0301607 & 0.090773 & 0.0106395 & 0.007366 & 0.046560 & 0.122890 \\
\hline
\end{tabular}




$$
\begin{aligned}
& \omega_{1}^{2}=-\lambda_{1,2}^{2}=\frac{-1+\sqrt{(1-36 \mu(1-\mu) b)}}{2}=\frac{1+M}{2} \\
& \omega_{2}^{2}=-\lambda_{3,4}^{2}=\frac{-1-\sqrt{1-36 \mu(1-\mu) b}}{2}=\frac{1-M}{2} \\
& \omega_{3}^{2}=-\lambda_{5,6}^{2}=1
\end{aligned}
$$

where $M=\sqrt{1-36 \mu(1-\mu) b}$.

The expressions (7) show that

$$
1>\omega_{1}>1 / \sqrt{2}>\omega_{2}>0
$$

and also it is clear that

$$
\omega_{1}^{2} \omega_{2}^{2}=9(1-\mu) b=1 / 4
$$

when $\mu=\mu_{0}$.

\section{ARNOLD'S THEOREM ON THE STABILITY AND THE EXISTENCE OF RESONANCES}

Since the characteristic equation of the linearised system has imaginary roots and the familtonian function (2) will not have a definite sign, so it is not possible to a ssert that the motion will be stable or unstable when all the terms of the familtonian function are taken into consideration. If the frequencies satisfy the condition

$$
0<\left|n_{1}\right|+\left|n_{2}\right|+\left|n_{3}\right| \leq 4
$$

then there exists (1927) a real canonical transformation $\left(q_{1}, p_{1}\right) \rightarrow\left(q_{1}^{\prime}, p_{1}^{\prime}\right)$, specified by power ser ies convergent in the neighbourhood of the origin such that the familtonian function (2) may be written as

$$
\mathrm{H}=\mathrm{H}^{\mathrm{O}}+\mathrm{H}^{\prime}\left(\mathrm{q}^{\prime}, \mathrm{p}^{\prime}\right)
$$

in the new variables, where $\mathbb{f}^{f}$ has the normal form

$$
\begin{aligned}
\mathrm{H}= & w_{1} r_{1}-w_{2} r_{2}+w_{3} r_{3}+c_{200} r_{1}^{2}+c_{110} r_{1} r_{2} \\
& +c_{101} r_{1} r_{3}+c_{020} r_{2}^{2}+c_{011} r_{2} r_{3}+c_{002} r_{3}^{2}
\end{aligned}
$$




$$
\left(2 r_{1}=q_{i}^{\prime 2}+p_{i}^{\prime 2}\right)
$$

and $\mathrm{H}^{\prime}$ is a convergent series in power sof $q_{1}^{\prime}, p_{1}^{\prime}$ beginning with terms not lower than the $f$ ifth one. he shall now a im to apply Arnold's theorem (1963) on the stability of the equilibrium position which is stated as follows:

Let the Hamiltonian function be such that

(a) the characteristic equation of the I inearised system has purely imaginary roots,

(k) the condition (8) is satisfied,

(c) the coefficient of the normal form (10) satisfy the inequality

$$
D=\left|\begin{array}{ll}
\frac{\partial^{2} H^{O}}{\partial r_{1} \partial r_{3}} & \frac{\partial H^{O}}{\partial r_{1}} \\
\frac{\partial H}{\partial r_{3}} & 0
\end{array}\right| \neq 0
$$

Then for most (in the sense of Lebesgue measure) initial conditions the equilibrium position

will be stable.

$$
q_{1}=p_{1}=0,
$$

During the present investigation, we a im to apply the above stated Arnold's theorem concerning the condition (8), we shall come across six resonance cases given as

$$
\omega_{1}-\omega_{2}=0 \text {, }
$$$$
\text { (ii) } \omega_{1}-2 \omega_{2}=0 \text {, }
$$$$
\text { (iii) } \omega_{1}-3 \omega_{2}=0,
$$

(iv) $2 \omega_{2} w_{3}=0$,

$$
\text { (v) } 3 \omega_{2}-\omega_{3}=0 \text {, }
$$$$
\text { (vi) } 2 w_{1}-w_{2} w_{3}=0 \text {. }
$$

In the adjoining figure 1 , we have plotted the values of $\mu$ corresponding to the different values of $b$ varying from 0 to 1 for all the six types of resonances. It has already been seen by kumar and Choudhry (1987) that within the range of stability given by the values of $\mu$ for $\omega_{1}=\omega_{2}$, which will be denoted as graph (i), we come across the resonances (iv), (v) and (vi) we find that the graph corresponding to the resonance (iv) lie within the graph (i) and the graphs for the (v) \& (vi) lie beyond. It shows that within the range of linear stability given by the graph (i), there is the possibility of having the resonances of the types (ii)-(iv). 
Since $\mathrm{H}_{3}$ and $\mathrm{H}_{4}$ are even functions of $\mathrm{q}_{3}$ and so after the normalisation $\phi_{3}$ will enter the arguments of sines and cosines as $2 \phi_{3}$ and $4 \phi_{3}$ and so corresponding to the cases (iv), (v) and (vi) we shall not have any critical case. so these cases need no special investigation. It is thus seen that except for (ii) and (iii) the Hamiltonian function can be reduced to the form ( 10$)$ suitable for the application of Arnold's theorem.

If the values of $u$ corresponding to $\omega_{1}=\omega_{2}$ be denoted

by $\mu^{\prime}$, then from the graph (i), it is clear that these values of $\mu_{0}^{0}$ will differ according to the different values of $b$.

From the equation (6) it follows that

$$
\omega_{1}^{2} \omega_{2}^{2}=1 / 4
$$

for $a l l b$ where $\omega_{1}=\omega_{2}$.

As in (1987) we shall restrict our investigation for the range of values of $\mu$ restricted to $0<\mu<\mu_{0}$ given by Table 1 where $\mu_{0}$ corresponds to $\omega_{1}-w_{2} w_{2}=0$ for the different values of $b$ and for such $\mu=\mu_{0}, \omega_{1}^{2} \omega_{2}^{2}=1 / 4$. Under such a restriction although the resonance (i) is avoided but we may come across the other resonance cases. As examined in (1972) the resonance relations (iv)-(vi) will not lead to the appearance of non ranishing denominators and they will not prevent the normal form ( 10$)$ from being obtained. So except for the two cases (ii) and (iii) the familtonian can be reduced to the normal form (10) required for the application of Armold's theorem. We shall need special consideration for the cases

(ii) and (iii).

5. NORMAL FORM OF THE HAMILTONIAN FUNCTION AND THE STABILITY EXCEPT FOR THE RESONANCE CASES (ii) \& (iii) WHERE $\mu<\mu_{0}$

Here ve shall aim to reduce the Hamiltonian function given by (2) to the form (10) for which we shall use Birkhoff's method of normalisation (1 985). If in the form (10), $\mathrm{H}_{2}^{(0)}$ is of positive definite form, then the equilibrium position is stable by virtue of Liapunov's theorem (1956) for all orders and all time. If $\mathrm{H}_{2}$ is not a function of definite sign, then we shall need the application of Arnold's theorem refer red above. In the present case $\mathrm{H}_{2}$ is not of definite form. To put $\mathrm{H}_{2}$ in the form ( 10$)$, we shall introduce the transfor mation referred in (1985), where we may ute 


$$
\begin{aligned}
& H=(1 / 2)\left(p_{1}^{\prime 2}+\omega_{1}^{2} q_{1}^{\prime 2}\right)-(1 / 2)\left(p_{2}^{\prime 2}+\omega_{2}^{2} q_{2}^{\prime 2}\right) \\
& +(1 / 2)\left(p_{3}^{\prime 2}+w_{3}^{2} q_{3}^{\prime 2}\right)+\sum_{\alpha+\beta=3}^{\infty} h_{\alpha \beta}{ }^{\prime \alpha} p^{\prime \beta} \\
& \alpha=\alpha_{1}+\alpha_{2}+\alpha_{3} \\
& B=B_{1}+\beta_{2}+B_{3}
\end{aligned}
$$

where for simplicity we shall mean

$$
\begin{aligned}
& h_{\alpha \beta}=h_{\alpha_{1} \alpha_{2} \alpha_{3} \beta_{1} \beta_{2} \beta_{3}} \\
& q^{\prime \alpha}=q^{\prime \alpha_{1}} q_{2}^{\prime \alpha_{2}} q_{3}^{\alpha_{3}} \\
& p^{\prime \beta}=q^{\prime \beta_{1}} p_{2}^{\beta_{2}} p_{3}^{\beta_{3}}
\end{aligned}
$$

and in subsequent investigations we shall not weite these ranges with the summation sign. Taking the notations as used in (1985) it may be mentioned that $h_{\text {g }}$ upto the third order and the fourth order terms not including $q_{3}^{\prime}$ and $p_{3}^{\prime}$ are given in (1985) which we shall not rewrite here. "The terms depend ing on $q_{3}^{\prime}$ and $p_{3}^{\prime}$ are $g$ iven here as follows:

$$
\begin{aligned}
& h_{102000}=a_{1}\left(H_{102000}+c_{1} H_{012000}\right) \text {, } \\
& \mathrm{h}_{012000}=\mathrm{a}_{2}\left(\mathrm{H}_{102000}+\mathrm{c}_{2} \mathrm{H}_{012000}\right) \text {, } \\
& h_{002100}=a_{1} b_{1} H_{012000} \\
& \mathrm{~h}_{002010}=-\mathrm{a}_{2} \mathrm{~b}_{2} \mathrm{H}_{012000^{\prime}} \\
& h_{202000}=a_{1}^{2}\left(H_{202000}+c_{1}^{2} H_{022000}+c_{1} H_{112000}\right) \text {, } \\
& h_{022000}=a_{2}^{2}\left(H_{202000}+c_{2}^{2} \mathrm{H}_{022000}+c_{2} H_{112000}\right) \text {, } \\
& h_{112000}=a_{1} a_{2}\left(2 H_{202000}+2 c_{1} c_{2} H_{022000}+\left(c_{1}+c_{2}\right) H_{112000}\right), \\
& h_{102100}=2 a_{1}^{2} b_{1} c_{1} H_{022000}+a_{1}^{2} b_{1} H_{112000} \\
& b_{102010}=-2 a_{1} a_{2} b_{2} c_{1} H_{022000}-a_{1} a_{2} b_{2} H_{112000}
\end{aligned}
$$




$$
\begin{aligned}
& h_{012100}=2 a_{1} a_{2} b_{1} c_{2} H_{022000}+a_{1} a_{2} b_{1} H_{112000^{\prime}} \\
& h_{012010}=-2 a_{2}^{2} b_{2} c_{2} H_{022000}-a_{2}^{2} b_{2} H_{112000^{\prime}} \\
& h_{002110}=-2 a_{1} a_{2} b_{1} b_{2} H_{022000^{\prime}} \\
& h_{004000}=H_{004000}
\end{aligned}
$$

Next we perform the following canonic transformations

$$
\begin{aligned}
& q_{1}^{\prime}=(1 / 2) q_{1}^{\prime \prime}+\left(i / \omega_{1}\right) p_{1}^{\prime \prime}, p_{1}^{\prime}=(1 / 2) i \omega_{1} q_{1}^{\prime \prime}+p_{1}^{\prime \prime}, \\
& q_{2}^{\prime}=-(1 / 2) i q_{2}^{\prime \prime}+\left(1 / \omega_{2}\right) p_{2}^{\prime \prime}, p_{2}^{\prime}=-(1 / 2) \omega_{2} q_{2}^{\prime \prime}+i p_{2}^{\prime \prime}, \\
& q_{3}^{\prime}=(1 / 2) q_{3}^{\prime \prime}+i p_{3}^{\prime \prime}, \quad p_{3}^{\prime}=(1 / 2) i q_{3}^{\prime \prime}+p_{3}^{\prime \prime}
\end{aligned}
$$
in the form

In the new variables the familtonian (12) may be witten

$$
\mathbf{H}=i \omega_{1} q_{1}^{\prime \prime} p_{1}^{\prime \prime}+i \omega_{2} q_{2}^{\prime \prime} p_{2}^{\prime \prime}+i \omega_{3} q_{3}^{\prime \prime} p_{3}^{\prime \prime}+\sum h_{\alpha \beta}^{\prime} q^{m \alpha} p^{m \beta}
$$

If $h_{\alpha \beta}^{\prime}=x_{\alpha \beta}+i y_{\alpha \beta}$ then the coefficients of terms not involving $q_{3}^{\prime}$ and $p_{3}^{\prime}$ are already given in (1987) which we shall not repeat here, except it may be noted that there we have to replace $h_{\alpha_{1} \alpha_{2} \beta_{1} \beta_{2}}$ by $h_{\alpha_{1} \alpha_{2}} \circ_{\beta_{1} \beta_{2}} \circ$ and similarly

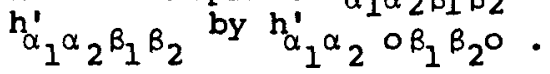

The coefficients with the terms involving $q_{3}^{\prime}$ and $p_{3}^{\prime}$ may be given as follows:

$$
\begin{aligned}
& x_{002100}=(1 / 4) h_{002100^{\prime}} \quad y_{002100}=\left(1 / 4 \omega_{1}\right) h_{102000^{\prime}} \\
& x_{000102}=-h_{002100^{\prime}} \quad y_{000102}=-\left(1 / \omega_{1}\right) h_{102000^{\prime}} \\
& \mathrm{x}_{001101}=-\left(1 / \mathrm{w}_{1}\right) \mathrm{h}_{102000^{\prime}} \mathrm{y}_{001101}=\mathrm{h}_{002100^{\prime}} \\
& \mathrm{x}_{002010}=\left(1 / 4 \omega_{2}\right) \mathrm{h}_{012000^{\prime}} \mathrm{y}_{002010}=(1 / 4) \mathrm{h}_{002010^{\prime}} \\
& x_{000012}=-\left(1 / \omega_{2}\right) h_{012000^{\prime}} y_{000012}=-h_{002010^{\prime}} \\
& x_{001011}=-h_{002010^{\prime}} \quad y_{001011}=\left(1 / \omega_{2}\right) h_{012000^{\prime}}
\end{aligned}
$$


the remaining coefficients of the third order terms involving $q_{3}^{\prime \prime}$ and $p_{3}^{\prime \prime}$ may be given by

$$
h_{\alpha \beta}^{\prime}=\left(y_{\alpha \beta}+i x_{\alpha \beta}\right)\left(-\omega_{1} / 2\right)\left(\beta_{1}-\alpha_{1}\right)\left(\omega_{2} / 2\right)\left(\beta_{2}-\alpha_{2}\right)\left(-\omega_{3} / 2\right)\left(\beta_{3}-\alpha_{3}\right)
$$

we shall firstly assume that the resonances of the types of (i)-(iii) are not present. As in (1987) we shall use Birkhoff's transformation

$$
\left(q_{3}^{\prime \prime}, p_{3}^{\prime \prime}\right) \longrightarrow\left(q_{3}^{m}, p_{3}^{m \prime \prime}\right)
$$

and nullify the third order terms.

The new familtonian exclusive of the third order and the fourth order terms giving rise to the resonance of type (iii) and al so those terms not necessary for the form (10) may be given as

$$
\begin{aligned}
H^{\prime}= & i w_{1} q_{1}^{\prime \prime \prime} p_{1}^{\prime \prime \prime}+i w_{2} q_{2}^{\prime \prime \prime} p_{2}^{m}+i w_{3} q_{3}^{m \prime} p_{3}^{\prime \prime \prime} \\
& -c_{200}\left(q_{1}^{\prime \prime \prime} p_{1}^{\prime \prime \prime}\right)^{2}+c_{110}\left(q_{1}^{m} p_{1}^{m}\right)\left(q_{2}^{m} p_{2}^{m}\right) \\
& -c_{002}\left(q_{3}^{\prime \prime \prime} p_{3}^{\prime \prime \prime}\right)^{2}+c_{101}\left(q_{1}^{m} p_{1}^{m}\right)\left(q_{3}^{\prime \prime \prime} p_{3}^{\prime \prime}\right) \\
& +c_{011}\left(q_{2}^{\prime \prime \prime} p_{2}^{\prime \prime}\right)\left(q_{3}^{\prime \prime} p_{3}^{\prime \prime}\right)-c_{020}\left(q_{2}^{m} p_{2}^{m}\right)^{2} \\
& +0\left(q_{1}^{2}+p_{1}^{2}\right)^{5 / 2}
\end{aligned}
$$

where

$$
\begin{aligned}
c_{200}= & -h_{200200}^{\prime}-(3 / 8) \omega_{1}^{2}\left(x_{000300}^{2}+y_{000300}^{2}\right) \\
& -(3 / 2)\left(x_{100200}^{2}+y_{100200}^{2}\right)+(1 / 2)\left(x_{100110}^{2}\right. \\
& +y_{100110}^{2}-\frac{\omega_{1}^{2}}{2 \omega_{2}\left(2 \omega_{1}-\omega_{2}\right)}\left(_{010200}^{2}+y_{010200}^{2}\right) \\
& +\frac{\omega_{1}^{2} \omega_{2}}{8\left(2 \omega_{1}+\omega_{2}\right)}\left(x_{000210}^{2}+y_{000210}^{2}\right)
\end{aligned}
$$




$$
\begin{aligned}
& c_{020}=-h_{020020}^{\prime}+(3 / 8) \omega_{2}^{2}\left(x_{000030}^{2}+y_{000030}^{2}\right) \\
& +\left(6 / \omega_{2}^{2}\right)\left(x_{020010}^{2}+y_{020010}^{2}\right) \\
& -\frac{\omega_{2}^{2}}{2 \omega_{1}\left(\omega_{1}-2 \omega_{2}\right)}\left(x_{100020}^{2}+y_{100020}^{2}\right) \\
& -(1 / 2)\left(x_{010110}^{2}+y_{010110}^{2}\right) \\
& -\frac{\omega_{1} \omega_{2}^{2}}{8\left(\omega_{1}+2 \omega_{2}\right)}\left(x_{000120}^{2}+y_{000120}^{2}\right) \text {, } \\
& c_{002}=-h_{002002}^{\prime}-\frac{2 \omega_{1}}{\omega_{1}-2}\left(x_{002100}^{2}+y_{002100}^{2}\right) \\
& -\frac{1}{8\left(w_{1}+2\right)}\left(x_{000102}^{2}+y_{000102}^{2}\right) \\
& \left.-(1 / 2) x_{001101}^{2}+y_{001101}^{2}\right)+\frac{2 \omega_{2}}{\omega_{2}-2}\left(X_{002010}^{2}+Y_{002010}^{2}\right) \\
& +\frac{\omega_{2}}{8\left(\omega_{2}+2\right)}\left(x_{000012}^{2}+y_{000012}^{2}\right) \\
& +(1 / 2)\left(x_{001011}^{2}+y_{001011}^{2}\right) \text {. } \\
& c_{110}=h_{110110}-\frac{2 \omega_{2}^{2}}{\omega_{1}\left(\omega_{1}-2 \omega_{2}\right)}\left(x_{100020}^{2}+y_{100020}^{2}\right) \\
& +\frac{\omega_{1} \omega_{2}^{2}}{2\left(\omega_{1}+2 \omega_{2}\right)}\left(x_{000120}^{2}+y_{000120}^{2}\right) \\
& -\frac{w_{1}^{2} w_{2}}{2\left(2 w_{1}+w_{2}\right)}\left(x_{000210}^{2}+y_{000210}^{2}\right)
\end{aligned}
$$




$$
\begin{aligned}
& -\frac{2 \omega_{1}^{2}}{\left(2 \omega_{1}-\omega_{2}\right) \omega_{2}}\left(x_{010200}^{2}+y_{010200}^{2}\right) \\
& +2\left(x_{010110} x_{100200}+y_{010110} y_{100200}\right) \\
& -\left(4 / w_{2}\right)\left(x_{020010} y_{100110}+x_{100110} y_{020010}\right), \\
& c_{101}=h_{101101}^{\prime}+2\left(x_{100200} x_{001101}+y_{100200} y_{001101}\right) \\
& \text { - }\left(x_{100110} x_{001011}+y_{100110} y_{001011}\right) \\
& -\frac{8 \omega_{1}}{\omega_{1}-2}\left(x_{002100}^{2}+y_{002100}^{2}\right) \\
& +\frac{w_{1}}{\left.2 w_{1}+2\right)}\left(x_{000102}^{2}+y_{000102}^{2}\right) \text {. } \\
& c_{011}=h_{011011}^{\prime}-2\left(x_{011001} x_{020010}+y_{011001} y_{020010}\right) \\
& +\left(x_{010110} x_{001101}+y_{010110} y_{001101}\right) \\
& -\frac{2}{2\left(\omega_{2}+2\right)}\left(x_{000012}^{2}+y_{000012}^{2}\right) \\
& +\frac{8 w_{2}}{\left(w_{2} 2^{-2}\right)}\left(x_{002010}^{2}+y_{002010}^{2}\right) \text {, } \\
& h_{200200}^{\prime}=-(3 / 2) \omega_{1}^{2} h_{000400}-\left(3 / 2 \omega_{1}^{2}\right) h_{400000^{-(1 / 2)}} h_{200200} \\
& h_{020020}^{\prime}=-(3 / 2) \omega_{2}^{2} h_{000040}-\left(3 / 2 \omega_{2}^{2}\right) h_{040000}-(1 / 2) h_{020020^{\prime}} \\
& h_{002002}^{\prime}=-(3 / 2) h_{00400} \\
& h_{110110}=\omega_{1} \omega_{2} h_{000220}+\left(1 / \omega_{1} \omega_{2}\right) h_{220000} \\
& +\left(w_{1} / \omega_{2} / h_{020200}+w_{2} / \omega_{1} / h_{200020^{\prime}}\right.
\end{aligned}
$$




$$
\begin{aligned}
& h_{101101}^{\prime}=-\left(1^{\prime} / \omega_{1}\right) h_{202000^{\prime}} \\
& h_{011011}^{\prime}=\left(1 / \omega_{2}\right) h_{202000^{\circ}}
\end{aligned}
$$

Passing now to the real variables introducing the transformation

$$
\left(q_{3}^{m}, p_{3}^{\prime \prime}\right)+\left(\overline{q_{3}}, \overline{p_{3}}\right)
$$

given by

$$
\begin{aligned}
& q_{1}^{\prime \prime}=\left(1 / \sqrt{\omega_{1}}\right)\left(\overline{q_{1}}-i p_{1}\right), p_{1}^{\prime \prime}=\left(\sqrt{\omega_{1}} / 2\right)\left(-\overline{i q_{1}} \mp p_{1}\right), \\
& q_{2}^{\prime \prime \prime}=\left(1 / \sqrt{\omega_{2}}\right)\left(\overline{i q_{2}}-\overline{p_{2}}\right), p_{2}^{\prime \prime \prime}=\left(\sqrt{\omega_{2}} / 2\right)\left(\overline{q_{2}}-\overline{i p_{2}}\right), \\
& q_{3}^{\prime \prime \prime}=\left(d / \sqrt{\omega_{3}}\right)\left(\overline{q_{3}}-i \overline{p_{3}}\right), p^{\prime \prime}=\left(\sqrt{\omega_{3}} / 2\right)\left(-i \overline{q_{3}}+\overline{p_{3}}\right)
\end{aligned}
$$

and then to polar co-ordinates

$\bar{q}_{3}=\sqrt{2 r_{3}} \sin \phi_{3}, \quad \bar{p}_{3}=\sqrt{2 r_{3}} \cos \phi_{3}$

we shall find that the lamiltonian (19) reduces to

$$
\begin{aligned}
\overline{\mathbf{B}}= & r_{1} \omega_{1}-r_{2} \omega_{2}+r_{3} \omega_{3}+(1 / 4)\left[c_{200} r_{1}^{2}+c_{020} r_{2}^{2}+c_{002} r_{3}^{2}\right. \\
& \left.+c_{110} r_{1} r_{2}+c_{101} r_{1} r_{3}+c_{011} r_{2} r_{3}\right]+o\left(r_{3}\right)^{5 / 2}
\end{aligned}
$$

Now to test regarding the stability of the equilibrium points under reference we shall examine the value of det $D$ given by

$$
\operatorname{det}\left|\begin{array}{ll}
\frac{\partial^{2} \bar{H}}{\partial r_{i} \partial r_{j}} & \frac{\partial \bar{H}}{\partial r_{i}} \\
\frac{\partial \bar{H}}{\partial r_{j}} & 0
\end{array}\right|_{r_{1}=r_{2}=r_{3}=0}
$$


on its expansion, we shall find that

$$
\begin{aligned}
16 D= & \omega_{1}^{2}\left(C_{011}^{2}-4 C_{020} C_{002}\right)+w_{2}^{2}\left(C_{101}^{2}-4 C_{200} C_{002}\right) \\
& +\omega_{3}^{2}\left(C_{110}^{2}-4 C_{200} c_{020}\right)-2 \omega_{1} \omega_{2}\left(C_{101} C_{011}+2 C_{002} C_{110}\right) \\
& -2 \omega_{1} \omega_{3}\left(C_{011} C_{110}+2 C_{020} C_{101}\right) \\
& -2 \omega_{2} \omega_{3}\left(C_{110} C_{101}+2 C_{200} C_{011}\right)
\end{aligned}
$$

After making some computations we may ind the coefficients given as

$$
\begin{aligned}
& c_{200}=\frac{\omega_{2}^{2}\left(124 \omega_{1}^{4}-696 \omega_{1}^{2}+81\right)}{144\left(1-2 \omega_{1}^{2}\right)^{2}\left(1-5 \omega_{1}^{2}\right)} \text {, } \\
& c_{110}=\frac{\omega_{1} \omega_{2}\left(64 \omega_{1}^{2} \omega_{2}^{2}+43\right)}{6\left(1-2 \omega_{1}^{2}\right)\left(1-2 \omega_{2}^{2}\right)\left(1-5 \omega_{1}^{2}\right)\left(1-5 \omega_{2}^{2}\right)} \text {. } \\
& c_{101}=\frac{-8 \omega_{1} \omega_{2}^{2}}{3\left(1-2 \omega_{1}^{2}\right)\left(4-\omega_{1}^{2}\right)} \\
& C_{020}=\frac{w_{1}^{2}\left(124 w_{2}^{4}-696 w_{2}^{2}+81\right)}{144\left(1-2 w_{2}^{2}\right)^{2}\left(1-5 w_{2}^{2}\right)} \\
& c_{011}=\frac{8 \omega_{2} \omega_{1}^{2}}{3\left(1-2 \omega_{2}^{2}\right)\left(4-\omega_{2}^{2}\right)} \\
& c_{002}=\frac{-\omega_{1}^{2} \omega_{2}^{2}}{3\left(4-\omega_{1}^{2}\right)\left(4-w_{2}^{2}\right)}
\end{aligned}
$$

Which coincide with those of Markeev (1 972). Putting $u=\omega_{1}^{-2} \omega_{2}^{-2}$, we find that (25), on substitutions of the values for the co-efficients $c_{i j k}$ given by $(26)$, may be
witten as 


$$
16 D=\frac{f(u)}{5184(4-u)^{2}(25-4 u)^{2}(1+12 u)^{2}}
$$

where $f(u)=73908288 u^{5}-356526576 u^{4}+2645643564 u^{3}$

$$
\text { - } 5787985485 u^{2}-759408680 u-317395600
$$

and for $u=4$,

$$
f^{\prime} f^{\prime}, f^{\prime}, f^{m}, f^{i v}, f^{v}
$$

are all positive where dashes denote the differentiations. Hence by Newton's theorem (Burnside and Panton, 1979) on the superior 1 imits of the roots it follows that there will be no root for $u>4$, whence it follows that $D \neq 0$ for such a restriction.

In Markeev's ca se when $\mu=\mu, u=4$, but in our case $u=4$ for all $\mu=\mu_{0}$ correspond ing to the case $\omega_{1}-\omega_{2}=0$ plotted in our graph - (i). Thus we find that for each b, we shall have $d$ ifferent $\mu$. For example, when $b=0.20$ the motion will be stable for all $\mu<0.1666666$ and so we find that corresponding to the different values of the pair $\left(\delta_{1}, \delta_{2}\right)$ our range of stability will go on differing.

$$
\text { Since } \mu_{0}=0.0285454 \text { is less than all the values of } \mu_{0}
$$
in the Table-l, so the motion will be stable for all $\mu<\mu_{\circ}$
but it leaves many values of $\mu$ for which al so Armold's theorem will hold and consequently the stability will hold except for the two resonance cases who se corresponding values of $\mu$ are all less than $\mu$ for each $b$. So the investigation of the stability for the resonance cases cannot be escaped.

\section{STABILITY FOR THE RESONANCE CASES (ii) AND (iii):}

(a) The resonance case (ii) $\omega_{1}-2 w_{2}=0$ :

As in (Kumar \& Choudhry, 1988), we shall introduce the transformation (18) to the Hamiltonian (15) but now we retain the terms giving $r$ ise to the resonance case and we shall finally have

$$
\begin{aligned}
\mathrm{H}^{\prime}= & i w_{1} q_{1}^{\prime m \prime} p_{1}^{\prime \prime \prime}+i w_{2} q_{2}^{\prime \prime \prime} p_{2}^{\prime \prime \prime}+i w_{3} q_{3}^{\prime \prime \prime} p_{3}^{m}+h_{100020}^{\prime} q_{1}^{m} \\
& p^{m}{ }^{2}+h_{020100}^{\prime} q_{2}^{\prime \prime 2} p_{1}^{m}+
\end{aligned}
$$


Passing now to real variables by means of the transformation (21), our Hamiltonian (28) reduces to

$$
\begin{aligned}
\mathbf{H}^{\prime}= & 2 \omega_{2} r_{1}-\omega_{2} r_{2}+r_{3}-\sqrt{\omega_{2}\left(x_{100020}^{2}+y_{100020}^{2}\right) r_{2} \sqrt{r_{1}}} \\
& \times \sin \left(\phi_{1}+2 \phi_{2}\right)+\tilde{H}\left(r_{j} \phi_{j}\right)
\end{aligned}
$$

where $\tilde{H}$ has the period $2 \pi$ in $\phi_{j}$ and $\tilde{H}=0\left(r_{1}+r_{2}\right)^{2}$. If $x_{100020}^{2}+y_{100020}^{2} \neq 0$ the equilibrium point will be unstable by Markeev's theorem (1978).

It has been examined in the paper (Kumar \& Choudhry, 1988 ) that $x_{100020}^{2}+y_{100020}^{2}$ which is the same as $(\delta)$ in Table II of the said paper is not zero for the region under consideration and its shows that the motion will be unstable.

(b) The resonance case $\omega_{1}=3 w_{2}$

In this case proceeding similar as in the paper (1988) which we shall not rewrite here, the familtonian may be reduced to

$$
\begin{aligned}
H= & 3 w_{2} r_{1}-w_{2} r_{2}+r_{3}+c_{200} r_{1}^{2}+c_{110{ }_{1} r_{2}}+c_{101} r_{1} r_{3} \\
& +c_{011} r_{2} r_{3}+c_{020} r_{2}^{2}+c_{002} r_{3}^{2} \\
& \left.+(1 / 3) w_{2} \sqrt{3\left(x_{100030}^{2}+y_{100030}^{2}\right.}\right) \times r_{2} \sqrt{r_{1} r_{2}} \cos \left(\phi_{1}+3 \phi_{2}\right) \\
& +0\left(r_{1}+r_{2}\right)^{5 / 2} \\
\text { Deroting by } & \\
a & =c_{200}+3 c_{110}+9 c_{020} \\
d & =3 w_{2} \sqrt{x_{100030}^{2}}+y_{100030}^{2}
\end{aligned}
$$

it is known by Markeev's theorem (1978) that if $|a|<d$, the equilibrium position is unstable and if $|a|>d$, the equilibrium position is stable. 
If $a=0$, the consideration of higher order terms becomes necessary.

The values of $a, d$ and the corresponding nature of the motion have already been computed in Table III of the refer$r$ ed work (1988). So here even in the thiree-dimensional ca se the nature will continue to be the same and it will not be rewe itten.

\section{CONCLUSIONS}

Thus we have shown that the triangular solution of the three-dimensional photo-gravitational circular restricted three-body problem is stable, for most sufficiently mall initial departures from the given solution except for the two resonance cases $\omega_{1}=2 \omega_{2}$ and $\omega_{1}=3 \omega_{2}$ and the range $\mu<\mu_{0}$. Under the resonance case $\omega_{1}=2 \omega_{2}$, the motion is seen to be unstable and for $\omega_{1}=3 \omega_{2}$ for some sets of values of the pair $\left(\delta_{1}, \delta_{2}\right)$ the motion is stable and for others it is unstable. These values are given in the referred paper (1988).

\section{REFERENCES}

[1] Arnold, V.I. (1 963), U SP.Matem. Nauk., Vol.18, pp.86-1 01 .

[2] Birkhoff, G.D. (1 927), Dymamical Systems, Am. Math. Soc.

[3] Burnside, W.S. and Panton, A.W. (1 979), Theory of Equations, S.Chand \& Company Ltd., New Delhi (l3th edition).

[4] Kumar, V. and Choudhry, R.K. (1 987), Cel. Mech.Vol.40, No.2, pp. 155-170.

[5] Kumax, V. a nd Choudhry, R.K. (1988), Cel. Mech.Vol. 41, No.2, pp. $161-173$.

[6] Liapunov, A.M. (1956), A General Problem of Stability of Motion, Collected Works, Vol.2, Acad. Sc., U SSR.

[7] Manju and Choudhy, R.K. (1 985), Cel. Mech.Vol.36, No.2, pp. $165-190$.

[8] Markeev, A.P. (1 972 ), Soviet Astronomy, Vol. 15, No. 4, Pp. $682-686$.

[9] Markeev, A.P. (1978), Liberation Points in Celestial Mechanics, and Cosmo-d ynamics, Moscow "Science". 